\title{
A WORKFLOW FOR UAV's INTEGRATION INTO A GEODESIGN PLATFORM
}

\author{
P. Anca ${ }^{\text {a }}$, A. Calugaru ${ }^{\text {a,b }}$, I. Alixandroae ${ }^{\text {b }}$, R. Nazarie ${ }^{\text {a* }}$ \\ a Esri, Bucharest, Romania, esri@esriro.ro \\ ${ }^{\mathrm{b}}$ Faculty of Land Reclamation and Environmental Engineering
}

Commission Th11

KEY WORDS: UAV,geodesign, 3Dmodelling, accuracy

\begin{abstract}
:
This paper presents a workflow for the development of various Geodesign scenarios. The subject is important in the context of identifying patterns and designing solutions for a Smart City with optimized public transportation, efficient buildings, efficient utilities, recreational facilities a.s.o.. The workflow describes the procedures starting with acquiring data in the field, data processing, orthophoto generation, DTM generation, integration into a GIS platform and analyzing for a better support for Geodesign. Esri's City Engine is used mostly for 3D modeling capabilities that enable the user to obtain 3D realistic models. The workflow uses as inputs information extracted from images acquired using UAVs technologies, namely eBee, existing 2D GIS geodatabases, and a set of CGA rules. The method that we used further, is called procedural modeling, and uses rules in order to extrude buildings, the street network, parcel zoning and side details, based on the initial attributes from the geodatabase. The resulted products are various scenarios for redesigning, for analyzing new exploitation sites. Finally, these scenarios can be published as interactive web scenes for internal, groups or pubic consultation. In this way, problems like the impact of new constructions being build, re-arranging green spaces or changing routes for public transportation, etc. are revealed through impact and visibility analysis or shadowing analysis and are brought to the citizen's attention. This leads to better decisions.
\end{abstract}

\section{INTRODUCTION}

The use of unmanned aerial vehicles in the field of Geographic Information Systems, has considerably reduced the time for acquiring data. In addition to this, the collected data in the form of georeferenced aerial imagery had benefit of improved resolution of up to $1.5 \mathrm{~cm}$. Tasks involving weeks of field work, can be achieved in just one day with only a small team of people. This not only translates into effective management of resources, but also in additional time for processing and analyzing images taken. The highly accurate results from the proceed data can be further integrated in dedicated 3D modelling softwares, such as Esri's CityEngine, serving as terrain elevation models and textures, and as extrusion height for different urban features.

This paper aims to present how ArcGIS proves to be a Solutions Plaform that assists GIS professionals, stakeholders and the populations affected to engage in "what if" type of activities and see the results of different development scenarios rapidly.

We combined sketching and modeling tools with the power of geo-data, by including $2 \mathrm{D}$ and $3 \mathrm{D}$ creative site analysis, sketching, diagramming and 3D procedural modeling as part of the design process.

\section{DATA ACQUISITION}

Data acquisition has been done using eBee from senseFly. This platform is a professional mapping drone used to capture highresolution aerial photos. Once we captured accurate aerial imagery, we then processed and transformed them into $2 \mathrm{D}$ orthomosaic and 3D models.

Our study area is situated in a peripheral commune in the northwestern part of Bucharest, called Mogosoaia.

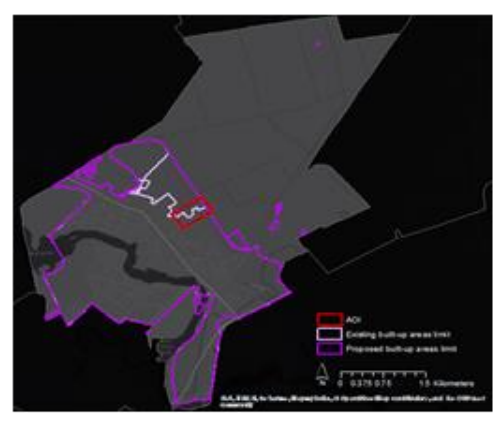

Figure 1 . The study area in the Mogosoaia comune For these area, in less than 40 minutes we have collected 347 geo-referenced images. We processed the raster data and delivered the final products in order to used them in the geodesign process.

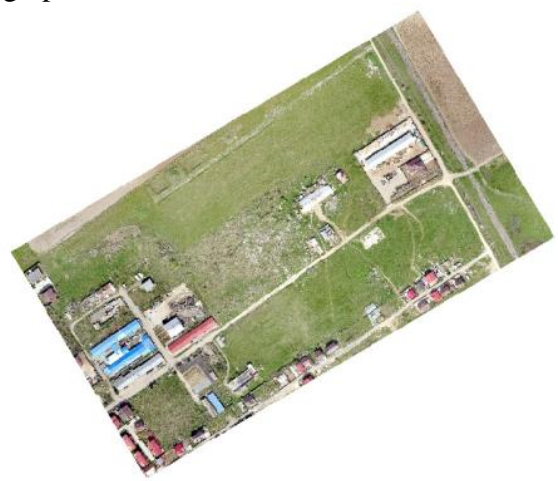

Figure 2. Orthophoto

\footnotetext{
* First author
} 


\section{DATA PROCESSING}

\subsection{Geodesign process}

Simply put, Geodesign is designing with ,geo-data”, while encouraging participation in the design process and creating greater understanding of the complex issues being designed out for those populations affected by the final outcome. (https://www.gislounge.com/)

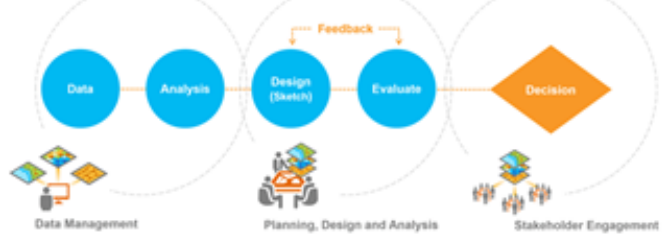

Figure 3. Steps in the process of Geodesign

While administratively independent from the Capital, Mogosoaia has strong economic and social ties with it: many of its inhabitant's work, study and spend their spare time in Bucharest. Last year, the local authorities have launched a complex project of developping the Commune Development Strategy and updating the General Urban Plan documentation.

Taking in consideration all these aspects, it will be interesting to follow the evolution that Mogosoaia will have in relation with Bucharest, whether it continues to be a ,satellite-commune" or it would detach easily, trying to assert economically, educationally aso.

While inside the administrative boundary of Mogosoaia, the study area is situated on the both existing and proposed built-up areas limit. Considering the land use pattern in the neighbourhood and the tendencies of pushing the built-up area limit toward the land reserve in the northern part of the commune, we proposed a scenario in which the study area will have a residential function.

The factors that we took into account in making the proposals for the urban morphology of the new residential zone are were: the accessibility to the main public services in the area, the configuration of the street network and the cadastre.

As for the first factor, we were interested to see the time needed for a walking person to arrive at the school, hospital, park and shops in the area.

For this analysis we made use of the Network Analyst extension capabilities. First we build a Network Dataset from the existing street network, with one of the costs being the pedestrian time (=the average per hour for a walking person), considered with a speed of $3 \mathrm{~km} / \mathrm{h}$. Next set the Service Areas for 5 to 20 minutes of walking. The results of the analysis are shown below: if the accessibility of the new residential area is quite good for the hospital, school and shops, the analysis for the only existing park in the commune shows the need of enriching the green infrastructure in the area.

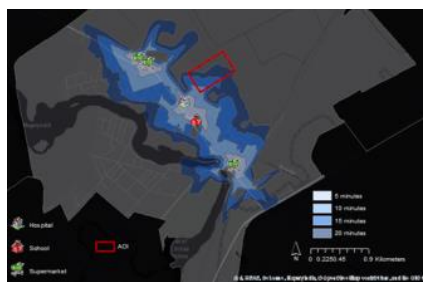

Figure 4. Accessibility of the study area to the existing public services

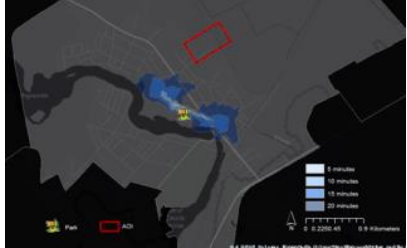

Figure 5. Accessibility of the study area to the existing park

Moving forward, the next factor studied is the street network. First we analysed the actual configuration of the street network making use of both vector an raster data. The raster data was generated using the Line Density tool, from the Spatial Analyst extension, having as input the street network in vector format.

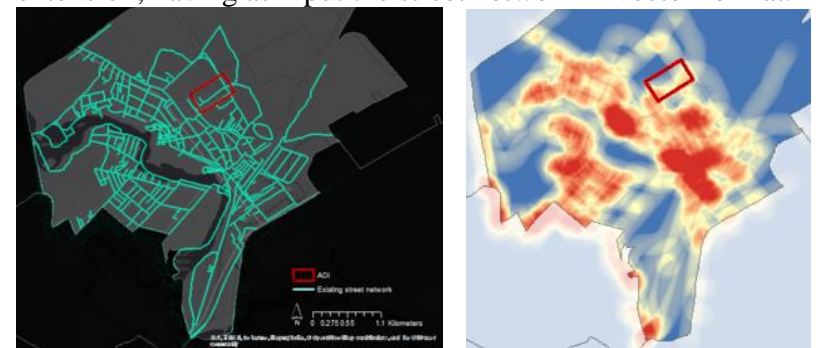

Figure 6 (a)Existing network

b) Density of the existing network
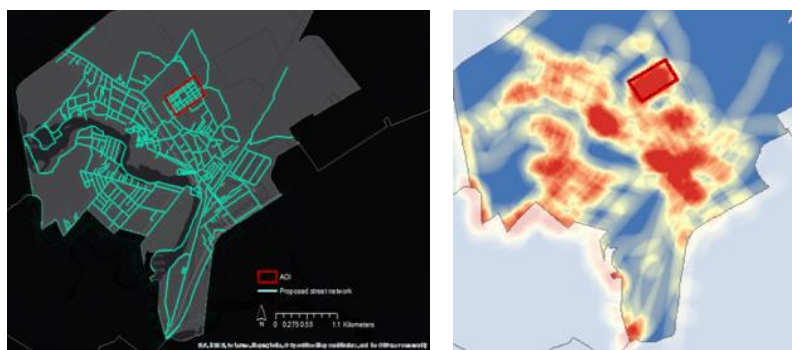

(a)Proposed network

(b) Density of the Proposed network

After making the proposals for the configuration of the street network in the study area, the raster analysis indicates a high density of the proposed network, which can be taken in consideration when further planning the street network for the rest of the neighbourhoods.

The new street network also indicates the necessity for reconsidering the actual cadastre in the study area, in order to optimize the land use.

Getting outside of ArcMap, we move our analyse in GeoPlanner, a WEB-GIS application, based on ArcGIS Online, which offers the necessary tools in order to compare multiple scenarios and decide which one suites best the vision and local strategy of the authorities, people of the place and stakeholders (Carl Steinitz, A framework for Geodesign)

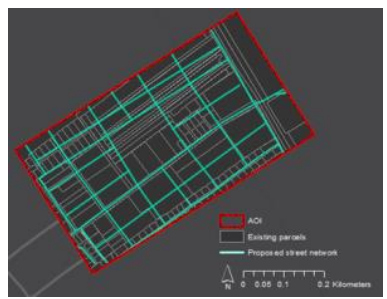

Figure 7. Geodesign

In the „Evaluate” section of the application, the user is provided with the necessary tools to compare two scenarios both visual and statistical. On the left side we can see the zoning in the existing conditions, while the right side shows the proposals for 
the new zoning. The Dashboards offers information regarding the surface of each land-use type. Helped by these tools, the actors involved in the process of redesigning the new residential area can make informed decision, comparing the existing conditions and the proposals with the local development strategy.

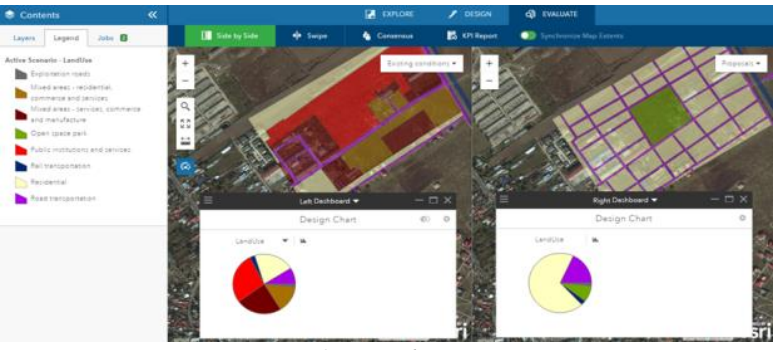

Figure 8. Geodesign process

\subsection{D Modelling}

Procedural modelling is a term for a certain number of techniques in computer graphics to create $3 \mathrm{D}$ models and textures from sets of rules. ESRI CityEngine uses a procedural modeling approach to automatically generate models through a predefined rule set. The rules are defined through a CGA shape grammar system enabling the creation of complex parametric models. Users can change or add the shape grammar as much as needed providing room for new designs. Due to the modelling technology, all buildings can be made to vary from one another to achieve an urban aesthetic (Mueller, P., et all, 2006, Alexander, C., et all, 1977, Blumberg, B., M., et all, 1995, Catmull, E., et all, 1978, Erbert, D., et all, 1998).

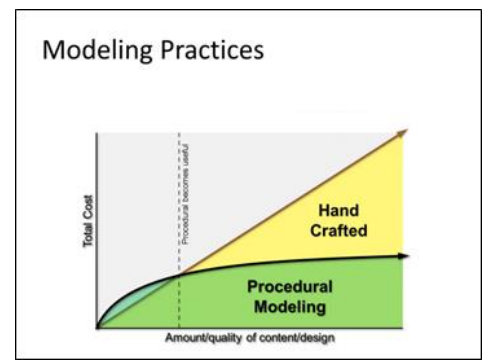

Figure 9. Grammar-based Modelling

The idea of grammar-based modeling is to define rules that iteratively refine a design by creating mode and mode detail. These rules operate on shapes which consist of a geometry in a locally oriented bounding-box. The following rule derivation illustrates the process: on the left side the initial shape is shown and on the right side the resulting generated model is displayed (CityEngine Overview, www.ce.help.esri.com).

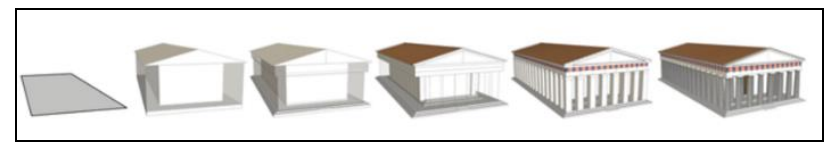

Figure 10. Example of 3D model created using CGA rules

The data gathered was processed in ArcMap application from ESRIs ArcGIS for Desktop, where it was organized by layers, each one of it representing real information from that area.
The data was also stored and managed into a geodatabase, a specific spatial database which is $100 \%$ interoperable with CityEngine application; this way, data transition was very fast and effective.

It was organized in layers for showing different design condition in the analysed area; the imagery obtained from the UAV was used as a support for the 3D models which were generated from the GIS vector data extracted in ArcMap application based on the resulted observations.

CGA grammar rules were used for transforming 2D GIS data into 3D models with real properties; the rules allowed the creation of different scenarios for both zoning and design in the specified area and also offered a new perspective for visualizing and analysing the data.

The polyline GIS data was imported as street graphs and the polygons as shapes with real properties and real position due to the specified coordinate system.

A zoning rule was applied on the zoning layers and allowed the creation of 3D zoning models with different colours for different types and with different heights for simulation of height restrictions in the area. This way, the design process is more intuitive for the generation of buildings, streets and constructions.

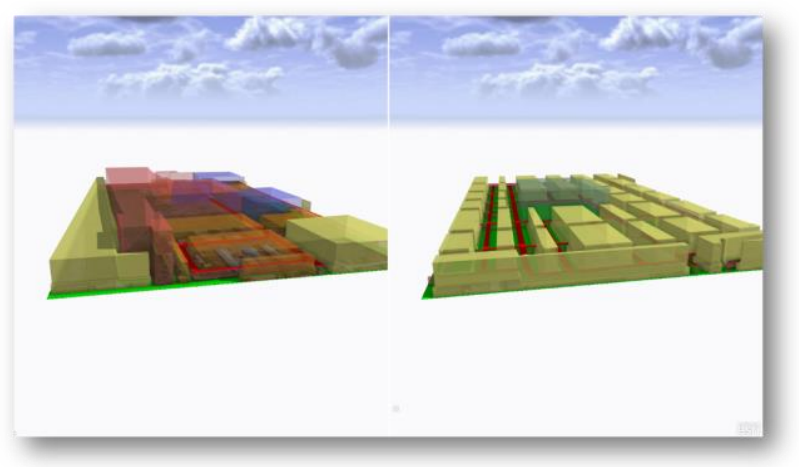

Figure 11. Comparison view for the zoning scenarios

The next step was to use another set of rules for creating the street models and buildings; the design had to be iterative for achieving two different scenarios.

Generation of the existing design meant the process of creating the existing types of buildings in the area and the resemblance. The models had to be framed into the existing landscape and for obtaining the real height of the buildings was used an industrial type of CGA rule which managed to generate a similitude between real landscape the scenario.

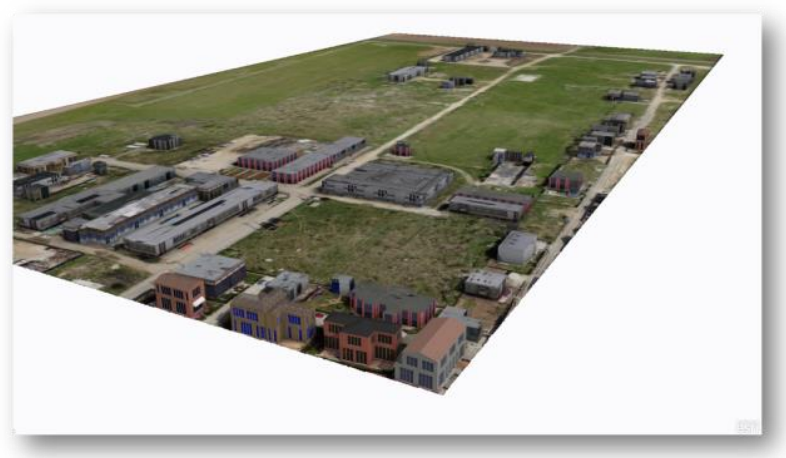

Figure 12. Simulation of the existing design 
After the existing design was simulated and checked for properness in the existing zoning conditions, the next step was to use the data for the proposed scenario for generating the $3 \mathrm{D}$ models.

The proposed design had to match the proposed zoning rules and also to simulate the landscape. Two CGA rules were used for creating the street models and the buildings.

The specification was to simulate the existence of modern streets with different number of lanes, with special lanes for public transportation and for bikers, with different forms of street objects like lamps at a specified distance between them, traffic lights, bus stops or bike racks, with vegetation for environmental reasons and pollution decrease.

The CGA rule for the street graph allowed the creation of the street models with all the conditions and specifications mentioned above, and also permitted a control of the entire network, not only a desired section. The $3 \mathrm{D}$ generation process was almost instantaneous and offered a new perspective of the design process.

After the street network models were generated, the buildings had to be framed into the landscape. For that, a designated rule was used which permitted a control over the entire area and for the entire number of buildings. Special specifications and customizations were used to generate the models, like setting a minimum and a maximum height for the constructions, a setback from the sidewalks or between them, a vertical setback, different construction materials, different types of windows, and different forms of roofs with the possibility of installing photovoltaic panels. Over 40 custom specification could be set for a real simulation and for obtaining different building models but completely integrated.

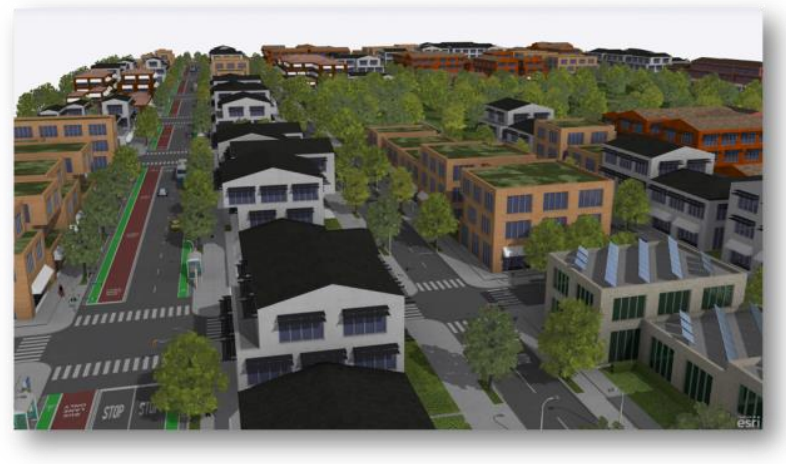

Figure 13. Simulation of the proposed design

The final steps after setting up the entire scene with all scenarios and representations were to upload the interactive 3D scene into the cloud for public access and to mark some bookmark into some locations of interest.

Because the used platform is completely integrated, the transition between 2D GIS application and data to the 3D GIS application and models and finally to the interactive 3D scene in the cloud was very easily accomplished.

The access is offered by a URL which is accessed from a browser with WebGL support. The scene will immediately load into the browser and interactive access to the models and information is gained. Also, the number of persons who can access the 3D web scene is unlimited, each one of them having the possibility to view different parts or locations from the scene.

Real analyzes are offered directly in the browser, like shadow analysis by choosing the time zone, the hour and month of the year. Also, real information is obtained if the user clicks a model: a house, a segment of the street or the sidewalk, a parcel or a defined portion of green space from the park model. The information is then visible in the right part of the webscene viewer, with different type of reports like: building performance, building consumption, water consumption per year, heating consumption per floor per year, footprint area, solar energy generated, grass area and so on. These reports are very important when designing different scenarios with real specification and conditions and being able to obtain them in such and interactive and fast method is one of the many strong capabilities the application can offer.

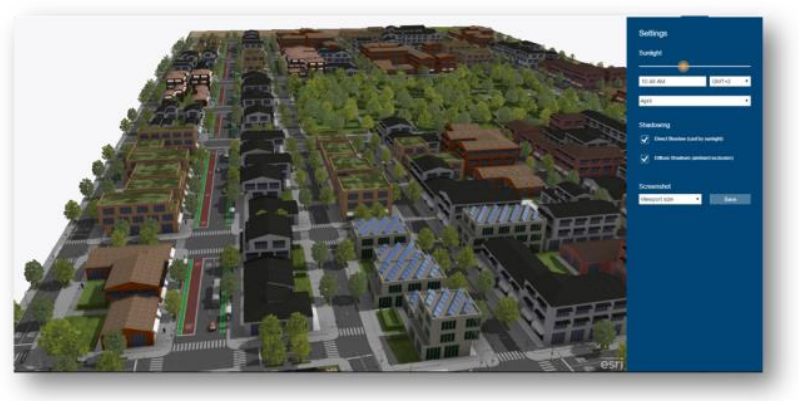

Figure 14. Simulation of shadow analysis

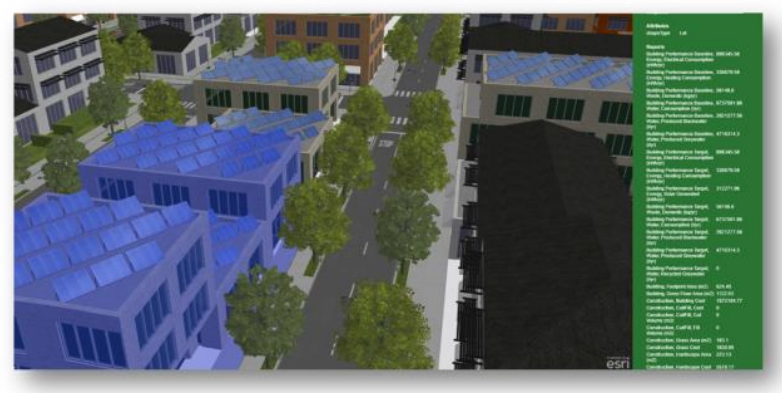

Figure 15. Reports for the selected models

The webscene viewer allows the users to view different locations by simply pressing a "play" button which triggers a transition effect between different bookmarks set in the scene. Also, anyone can control the models and their visibility by choosing whether the layers which is representative is on or off Besides that, by being accessed through an URL, that means that the scene could be embedded into web pages or sites and also could be viewed from PCs and laptops to smartphones and tablets.

By using modern platforms and technologies, the design process is more intuitive, more controlled and by far a better method for taking better decisions. Using an integrated platform that offers $100 \%$ interoperability between different applications and checkpoint into the analysis is making the difference between a discontinuous data transition and progress and having a rapid and straight forward workflow for integrating different data from different sources into one geodesign platform.

\section{CONCLUSIONS}

UAV technology has improved a lot in the last years. We now have access to unmanned devices that can be equipped with sensors in order to acquire data from above the ground. By further processing this data, and analysing with the help of specialized tool, we are able to find solutions to the daily problems of our society. The Mogosoaia Project is a clear example of how an integrated work flow for Geo - design works. Moreover, the fact that the results of the analysis are presented in the form of $3 \mathrm{D}$ web scenes and are realistic 
representation of how the area might look tomorrow, brings the community at the round table for discussions. This is an important step in involving the civil society in the decision making process and also, opens a subject for the future: Smart Cities.

\section{REFERENCES}

https://www.gislounge.com/

CityEngine Overview, www.ce.help.esri.com

Carl Steinitz, A framework for Geodesign,, Esri Press

Mueller, P., et all, 2006, Alexander, C., et all, 1977, Blumberg, B., M., et all, 1995, Catmull, E., et all, 1978, Erbert, D., et all, 1998)

Neitzel, F., 2011. Mobile 3D Mapping with a Low-cost UAV System. International Archives of the Photogrammetry, Remote Sensing and Spatial Information Sciences, Zurich, Switzerland, Vol. XXXVIII-1/C22. 